\title{
Téoros
}

Revue de recherche en tourisme

\section{L’aventure ? Casse-cou!}

\section{André Hut}

Volume 13, numéro 3, automne 1994

Le tourisme d'aventure : vers la maturité ?

URI : https://id.erudit.org/iderudit/1077113ar

DOI : https://doi.org/10.7202/1077113ar

Aller au sommaire du numéro

\section{Éditeur(s)}

Université du Québec à Montréal

\section{ISSN}

0712-8657 (imprimé)

1923-2705 (numérique)

Découvrir la revue

Citer cet article

Hut, A. (1994). L'aventure ? Casse-cou! Téoros, 13(3), 40-42. https://doi.org/10.7202/1077113ar

Ce document est protégé par la loi sur le droit d'auteur. L'utilisation des services d'Érudit (y compris la reproduction) est assujettie à sa politique d'utilisation que vous pouvez consulter en ligne.

https://apropos.erudit.org/fr/usagers/politique-dutilisation/
Cet article est diffusé et préservé par Érudit.

Érudit est un consortium interuniversitaire sans but lucratif composé de l'Université de Montréal, l'Université Laval et l'Université du Québec à Montréal. Il a pour mission la promotion et la valorisation de la recherche. https://www.erudit.org/fr/ 


\section{L'aventure? Casse-cou!}

André Hut

Tout déplacement touristique ne comprend-il pas une composante d'aventure? Et qualifié comme tel, jusqu'où et à quelles conditions peut-il s'accomplir?

\section{Le grain de sable}

Mềme si celui-ci a été méticuleusement préparé ou se détoule au sein d'un voyage organisé et planifié, nul n'est exempt des caprices du dimat pluvieux ou torride qui entraîne inondations, coupure de route ou de voies ferrées, comme aussi sécheresse, feux de forêt, rationnement d'eau et invasion d'insectes ou de méduses!

Qui peut prévoir que les aiguilleurs du ciel, les cheminots ou les douaniers wont se mettre en grève, que les transporteurs routiers ou les agriculteurs vont bloquer les routes? Des travailleurs en empêchent d'autres, contre leur gré, de jouir de leurs congés payés et loisir's pour sauver ou améliorer leur droit au travail, conditions de salaire ou organisation des tâches! Les usagers ou consommateurs sont pris en otages par les prestataires des services ou les producteurs afin de forcer les pouwoirs économiques et/ou politiques à plicr et négocier pour éviter l'impopularité et la pagaille!

En conséquence, pour les uns, aux déjà pénibles heures de patience au sein des longues files de voitures ralenties par les bouchons classiques, sétirant sur des dizaines de kilomètres, s'ajoutent l'inattendue désorganisation de l'itinéraire et des projets de haltes nocturnes, le manque de boissons et de provisions, le tout sous le soleil caniculaire; pour d'autres, le dérèglement des correspondances, l'enfermement dans le stress et l'incertitude de l'aéroport, la promiscuité de la foule, la pénurie des magasins dévalisés, la claustrophobie accentuée par la chaleur et l'inquiétude, les crises de nerfs et de pleurs alternant avec les coups de colère ou de

Monsieur André Hut est, de la Belgique, le correspondant régulier de Téor os pour l'Europer

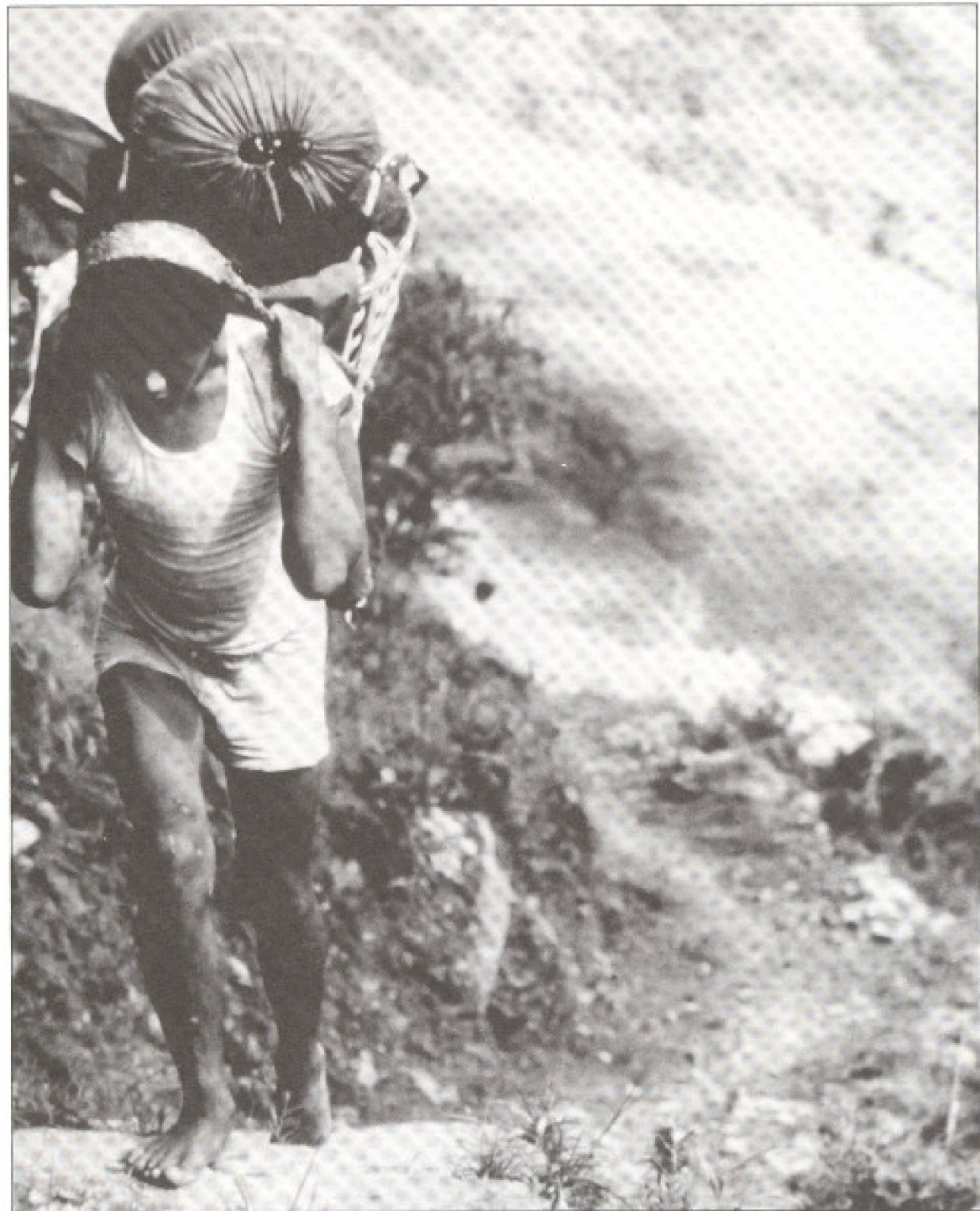

Nếp: Club Aventure.

déprime? L'aventure tourne au cauchemar!

Si la stratégie des grévistes ou des manifestants se révele souvent payante, on est en droit de s'interroger si le choix de ces moyens peut se défendre sur le plan d'une éthique sociale, compte tenu des wâts économiques et sociaux que les contestataires font payer auxusagersetà la collectivitédes citoyens.

\section{Des pratiques fondatrices}

Les premiers touristes, à la fin du XIX ${ }^{\mathrm{c}}$ siècle, n'ont-ils pas risqué de partir dans l'inconnu, sans précédents ni expérience? Ces Anglais, par exemple, qui ont inau- 


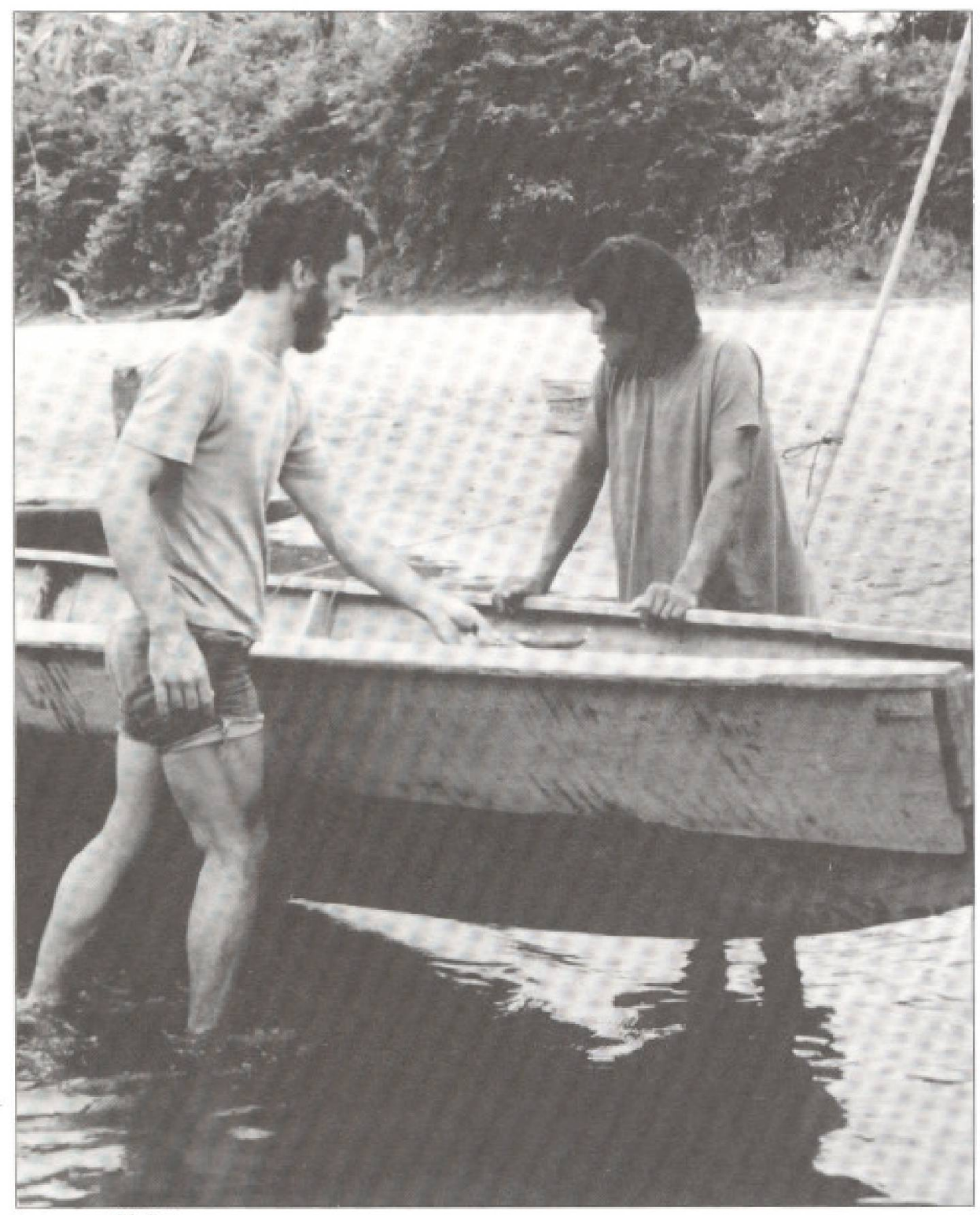

Amazone, Club Aventure.

guré, l'éré, les escalades dans les montagnes de France ou de Suisse, avec l'aide de guides locaux héréditairement aguerris? Ou les mêmes, l'hiver, important des skis des pays nordiques pour s'élancer sur les pentes neigeuses? Certains aventuriersont affronté rapides, tourbillons et chutes, emportés par les eaux bouillonnantes et traîtres des grands flewver en cavales!

Si les uns ont été attirés par les sommets, les autres fascinés par les profondeurs, s'engagèrent dans le labyrinthe des grottes inviolées, en spéléologues débutants ou explorèrent les abysses des mers grâce aux balbutiements de la plongée sous-marine naissante; enfin, appelés par le large, certains n'ont-ils pas aménagé des séjours et stages de voile? de millions de citoyens du Nord de l'Europe descendant, la guerre terminée, vers le soleil des côtes françaises et espagnoles! Inexpérimentés, ils avaient tout à apprendré le choix judicieux de l'itinéraire et des villes d'étapes, du camping de résidence compte tenude sa localisation; mais aussi ladaptation à lavie sous tenteet au plein air, à la chaleur, aux habitudes et productions locales, aux voisins de diverses langues et comportements; enfin, la réaction critique et le discernement face à l'exploitation commerciale de leur naiveté par les autochtones.

Après plusieurs années de retour au même endroit, comparaison aidant, beaucoupont acquis une expérience libératrice leur permettant d'être davantage attentifs aux relations quis'enchevêtrententreles membres de la famille, à reconstruire, chaque fois, au gré de l'évolution de chacun de ceux-ci. II s'agit d'une exploration d'un autre ordre, appelant écoute et compréhension, partage et négociations, complémentarité et convivialité; mêmes exigences vis-à-vis des amis et connaissances du camping. Enfin, ces vacanciers devaient affronter la nouveauté de vivre et gérer un temps de 3 à 4 semaines exclusivement consacréa ux loisirs alternant repos, détente et découverte, notamment, de la région et population d'accueil. Endéfinitive, lecamuld'aventures existentielles, relationnelles et socioculturelles!

\section{Le vagabondage touristique}

Il est compréhensible que les jeunes aient quitté les vacances sédentaires de leurs parents et les grandes concentrations estivales afin d'en prendre le contre-pied. En effer, une convergence de facteurs expliquent l'engouement et la mode en faveur d'un nomadisme touristique: le prolongement des études, la démocratisation des transports aériens et ferroviaires contribuant à la naissance des organisations de tourisme universitaire, le mouvement hippie et la contestation culturelle et libertaire de $1968 \ldots$ contribuèrent à lancer les étudiants sur les routes, sans itinéraire précis, sur le poutce, au gré du bon vouloir des automobilistes et des routiers, dormantà la belle étoile, sur les plages et les places publiques. Cette détinstallation physique et cet éclatement dans l'espace autant que dans les esprits, engendrèrent l'aventure mystique des voyages initiatiques vers Katmandou ou les grands rassemblements musicaux, à base de fusion sociale et d'évasion hallucinogène. 
Les fameux Guides du routard permettaient de sauvegarder à la fois indépendance et souplesse tout en s'assurant des points de chute éventuels, sécuritaires et bon marchếs.

Trop fréquemment, ces enfants de riches, retrouvant, au retour, leur confort, se vantèrent d'avoir abusé, sans contrepartie, de la royale hospitalité offerte gracieusement par leurs très modestes hôtes, parfois au seuil de la pauvreté: la plupart n'avaient même pas conscience de l'irresponsabilité de leurs actes.

\section{L'exotisme organisé}

De leur côté, dix ans plus tard, des agences de voyages commerciales et non-marchandes, attentives aux nouveaux besoins, conçoivent et vendent aux jeunes adultes qu'ils sont devenus - professions bien rémunérées et déja à certains postes decadres - des expéditions therrapeutigues qui contrecarrent les effets négatifs de l'immobilité et du stress: traversće du désert, de la savane africaine ou de la jungle asiatique, en voitures $4 x 4$ ou en canot motorisé... s'inspirant de modèles médiatiques comne le Paris-Dakar, suffisamment mis en cause et critiqué à juste titre.

La prise de conscience écologique - favorisée par les filmset les discours du commandant Cousteau ou les reportages de Nicolas Hulot dans ses émissions Ushuaia par exemple - rend certaines associations plus attentives aux effets négatifs des modes de transport mentionnés ci-dessus: elles proposent de se déplacer à pied, au Népal, à dos de chameaux dans le désert ou en pirogue sur l'Amazone; ces offres, accessibles seulement à des privilégiés, capables deles payer, sont déjà plus respectueuses de l'environnement, du moins à ce niveau des moyens de locomotion.

Il n'empêche que des reproches sont de plus en plus formulés, par les populations d'accueil, aux uns et aux autres: accumulation des déchets le long ou aux haltes des étapes, consommation des réserves alimentaires limitées des familles réceptrices, surcharge progressive de l'environnement par des groupes de plus en plus nombreux, comportements inconscients et irrespectueux, voire choquants, des voyageurs, folklorisation de leur culture et larbinisation, création de nouveaux besoins - artificiels - de consommation par imita- tion du modèle prestigieux de leurs hôtes, incompréhension de l'écart entre la richesse des moyens déployés pour l'expédition et la modicité des aides acoordées par leurs pays d'origine pour le développement...

D'ailleurs, le tourisme d'aventure s'exerçant souvent dans des régions situées en dehors et à distance des sites touristiques classiques, les populations autochtones ne sontguèreencontactavecdes Occidentaux, peu préparées à la renoontre avec cette nouveauté et cette al térité, assez démunies pour gérer ces chocs et affrontements culturels.

En conséquence, des organismes engagés dans la coopération et le développernent ou l'éducation permanente, relayant les dénonciations et lestravers relatésci-dessus, invitent leurs membres et amis à passer de l'exotisme et des pseudo-alternatives à des initiatives qui privilégient, d'une manière dotue et en petits groupes, la rencontre et la cohabitation provisoire avec la population locale, au rythme de sa vie et activités quotidiennes. Par exemple: la visite de projets en cours dans le tiers-monde par des personnes qui les soutiennent et en rendront compte au retour, des échanges dejeunes du même mouvement dejeunesse - scoutisme, ruraux - ou d'élèves de deux écoles jumelées; en Europe, au pays, des traversées à pied ou à vélos de régions rurales et de parcs naturels en contact avec les autochtones, le long des sentiers de grandes randonnées, séjours au camping à la fermeou en chambres et tables d'hôteset même, pour certains, cheminement sur les routes de pèlerinages, vers Compostelle....

\section{Une éthique de l'aventure}

De cette histoire et ces expériences d'aventure touristique se dégagent progressivement despistes et des exigences en faveur d'une éthique de ce type de pratiques.

Il n'est plus désormais acceptable que le voyageur, d'une part, profite seul des avaniager que peuvent lui apporter ces démarches et, d'autre part, ne prenne pas conscience des effets négatifs et pervers qui en découlent au niveau de lenvironnement physique, socioculturel, économique et spirituel des populations locales où se déroule l'activité en question.
Il est inadmissible et condamnable de partir en escalade ou en ski hors-piste, en mer, dans le désert ou la jungle, sur un fleuve ou dans une grotte... sans tenir compteni de la météo ni des conseils judicieux de personnes expérimentées et compétentes. Il serait en effetsuicidaire d'entreprendre l'expédition sans bénéficier en outre des conditions de sécurité indispensables: un minimum d'initiation et d'entraînement, un matériel adéquat et fiable, un accompagnement qualifié.

À cause de l'inconscience et/ou de la fanfaronnade d'écervelés, malheureusement de plus en plus nombreux, les communautés locales doiventsupporter les frais de recherches et de secours, et même fréquemment, risquer la santé et la vie de leurs sauveteurs. Les coûts économiques et sociaux éventuels, occasionnés par l'activité, doivent être supportés ausoi par les voyageurs et ne pas en laisser l'entièreté sur le dos des populations d'accueil. Il faut rechercher les formules et réglementations juridiques, nationales et internationales, susceptibles decontraindre, selonleurdegré de responsabilité et sur base de l'enquête, tant le voyageur que l'organisation de l'activité, à contribuer auxcoûttsoccasionnés par une imprudence ou un accident fortuit, quitte à se retourner vers les pouvoirs publics du pays d'origine.

Dans le mểne sens, les équipements et services collectifs qui rendent possible l'expédition devraient être partiellement remboursés par les utilisateurs, à l'instar des taxes de séjours qui sont perçues dans les pays touristiques européens.

Enfin, la préparation personnelle de chacun des participants ainsi que celle du groupe lui-même, oonstituent la garantie que les rencontres et ếchanges seront enridhissants pour les deux parties en présence. Sinon, les inégalités économiques et l'aliénation culturelle en ressortiraient davantage encore renforcées. 